\title{
On the Viscous Boundary Layer near the Center of the Resistive Reconnection Region.
}

\author{
Dmitri A. Uzdensky and Russell M. Kulsrud \\ Princeton Plasma Physics Laboratory, P.O.Box 451, Princeton University, \\ Princeton, New Jersey 08543
}

(March 23, 1998)

This paper studies the behavior of the magnetic field near the center of the reconnection layer in the framework of two dimensional incompressible resistive magnetohydrodynamics with uniform resistivity in a steady state. Priest and Cowley have presented an argument [1] showing that when the viscosity $\nu$ is zero, the magnetic separatrices do not cross at a finite angle but osculate at the X-point. In the present paper it is shown that this conclusion is in fact not correct. First, some results of numerical simulations of the reconnection layer are presented. These results contradict the conclusions of Priest and Cowley. In order to explain this contradiction, an analytical theory for the neighborhood of the X-point is developed in the second part of the paper. It is found that, if the viscosity is exactly equal to zero, then one of the critical assumptions of the above mentioned argument, namely the assumption that the stream function $\Phi$ can be Taylor-expanded near the X-point, breaks down. In the case of small but finite viscosity a boundary layer analysis in the vicinity of the neutral point is carried out. Some of the higher derivatives of $\Phi$ become very large near the X-point, leading to a non-zero angle between the separatrices. As $\nu$ goes to zero, the boundary layer shrinks and one can see the emergence of the non-analytic logarithmic terms in the expansion of $\Phi$ in the outer region. The results of the boundary layer analysis are found to be in good agreement with the numerical simulations.

PACS number(s): 52.30.Jb, 95.30.Qd, 96.60.Rd 


\section{INTRODUCTION.}

There is a very well known argument concerning the behavior of magnetic field near the center of the reconnection layer in the framework of two dimensional steady state incompressible resistive MHD (magnetohydrodynamics) with uniform resistivity. This argument was first made by Priest and Cowley [1], Cowley [2], and then also repeated by Shivamoggi $[3,4]$ and Biskamp $[5,6]$. The main result of this argument is that in the absence of viscosity the magnetic field lines do not cross at a finite angle (as in Fig. 1) but osculate (Fig. 2) at the $\mathrm{X}$-point. In this paper we first reproduce this argument, and then show where and how it fails.

Following Biskamp [5,6], the argument goes as follows.

We consider the central part of the reconnection layer with small uniform resistivity and viscosity and assuming incompressibility. In a steady state, the plasma is described by the resistive MHD equations:

$$
\begin{gathered}
\eta \mathbf{j}=\mathbf{E}+\mathbf{v} \times \mathbf{B} \\
\mathbf{v} \cdot \nabla \mathbf{v}=-\nabla p+\mathbf{j} \times \mathbf{B}+\nu \nabla^{2} \mathbf{v}
\end{gathered}
$$

where the density is set equal to unity.

Considering only the two-dimensional problem, we introduce the magnetic flux function $\Psi(x, y)$ and the stream function $\Phi(x, y)$ :

$$
\begin{aligned}
& \mathbf{B}=\mathbf{e}_{z} \times \nabla \Psi \\
& \mathbf{v}=\mathbf{e}_{z} \times \nabla \Phi
\end{aligned}
$$

Then, the steady state Ohm law (1) can be written as

$$
\partial_{x} \Phi \partial_{y} \Psi-\partial_{y} \Phi \partial_{x} \Psi=\eta \nabla^{2} \Psi-E
$$

where $E \equiv E_{z}=$ const.

Upon taking the curl of the equation of motion (2), we get

$$
\partial_{x} \Phi \partial_{y} \omega-\partial_{y} \Phi \partial_{x} \omega=\partial_{x} \Psi \partial_{y} j-\partial_{y} \Psi \partial_{x} j+\nu \nabla^{2} \omega
$$


where $\omega=\nabla^{2} \Phi$ is the vorticity, and $j \equiv j_{z}=\nabla^{2} \Psi$ is the current density.

We consider the simplest possible geometry with up-down and right-left symmetry (see Fig. 1). The stagnation point of the flow coincides with the neutral point of the magnetic field, that is with the center of the current layer $(x=0, y=0)$. Because of the reflection symmetry, the magnetic flux function must be even, and the stream function must be odd with respect to both $x$ and $y$ axes. Then, in the vicinity of the neutral point we write the Taylor expansion for $\Psi(x, y)$ and $\Phi(x, y)$ in $x$ and $y$ as follows:

$$
\begin{gathered}
\Psi(x, y)=\sum_{m, n} \Psi_{2 m, 2 n} \frac{x^{2 m} y^{2 n}}{(2 m) !(2 n) !} \\
\Phi(x, y)=\sum_{m, n} \Phi_{2 m+1,2 n+1} \frac{x^{2 m+1} y^{2 n+1}}{(2 m+1) !(2 n+1) !}
\end{gathered}
$$

Since at the origin $\mathbf{B}=\mathbf{v}=0$, and hence $\eta j(0,0)=E$, Eq. (5) gives

$$
\eta\left(\Psi_{20}+\Psi_{02}\right)=E
$$

Differentiating Eq. (5) twice with respect to $x$ at the origin one obtains

$$
2 \Phi_{11} \Psi_{20}+\eta\left(\Psi_{40}+\Psi_{22}\right)=0
$$

and differentiating the same equation twice with respect to $y$ at the origin,

$$
2 \Phi_{11} \Psi_{02}-\eta\left(\Psi_{22}+\Psi_{04}\right)=0
$$

Differentiating Eq. (6) once with respect to $x$ and once with respect to $y$ at the origin gives

$$
-\Psi_{20}\left(\Psi_{22}+\Psi_{04}\right)+\Psi_{02}\left(\Psi_{40}+\Psi_{22}\right)=\nu\left(\Phi_{51}+2 \Phi_{33}+\Phi_{15}\right)
$$

which, by use of Eqs. (10) and (11), becomes

$$
-\frac{4}{\eta} \Phi_{11} \Psi_{20} \Psi_{02}=\nu\left(\Phi_{51}+2 \Phi_{33}+\Phi_{15}\right)
$$

Making use of this result Biskamp and others then consider the inviscous case $\nu=0$ and drop the LHS (the left hand side) of Eq. (13). Then, assuming $\Phi_{11} \neq 0$, i.e. stream lines 
forming hyperbolae, either $\Psi_{20}$ or $\Psi_{02}$ has to be zero. Choosing $\Psi_{20} \neq 0$, which corresponds to the current layer along the horizontal midplane $x=0$, one gets

$$
\Psi_{02}=0
$$

or $B_{x}(x=0, y)=-\partial \Psi / \partial x \sim y^{3}$. This implies an osculating configuration (see Fig. 2), which is clearly different from the X-point configuration (Fig. 1) characterized by $B_{x}(x=$ $0, y) \sim y$. It is then argued that this behavior indicates the inherent tendency to formation of long current sheets in a reconnecting resistive magnetized fluid.

While we agree that in general for reconnection to occur a very narrow current sheet has to be formed, we do not agree with the conclusion (14).

Our objection to this conclusion is based on the following observation. We note that the above argument is designed to work inside the resistive diffusion region, and therefore means that $\Psi_{02}=0$ exactly, even on a microscopic scale. The derivation leading to Eq. (14) is actually based upon the assumption that the stream function remains analytic and can be Taylor-expanded even in the limit of zero viscosity. In other words, one implicitly assumes that the higher order derivatives, e.g. $\Phi_{51}$, on the RHS (the right hand side) of Eq. (13) stay finite as $\nu \rightarrow 0$.

However, if the viscosity is set to zero from the very beginning, then the term containing the highest order $x$-derivative of vorticity in Eq. (6) is $v_{x} \partial \omega / \partial x$. The coefficient before this derivative vanishes on the midplane $x=0: v_{x}(x=0, y)=0$. Therefore, as far as the $x$-direction is concerned, $x=0$ is a regular singular point of the equation of motion without viscosity, and one should actually expect a non-analytic behavior of the stream function near $x=0$. Thus, the Taylor expansion (8) should fail in this limit.

Now, if one keeps the viscosity finite, then, of course, the stream function is analytic, because $x=0$ is a regular point in this case. However, by considering the limit of very small viscosity, $\nu \ll \eta$, one introduces a new small parameter (the viscosity), and therefore it is not surprising that a boundary layer develops near the line $x=0$. When $\nu \rightarrow 0$, the thickness $\delta$ of this boundary layer goes to zero, but some higher order derivatives (such as 
$\left.\Phi_{51}\right)$ inside the boundary layer become (as we will show) very large. As a result, the RHS of Eq. (13) stays finite, while each of the factors on the LHS approaches a finite value as $\nu \rightarrow 0$. In particular, $\Psi_{02}$ does not have to be zero, thus allowing the separatrices to cross at a non-zero angle. Outside of the boundary layer (i.e. as seen at the scales much larger than the boundary layer thickness $\delta$ ) one can neglect the viscosity, but can no longer assume that the stream function (and the magnetic flux function as well) has a regular behavior and can be expanded in a Taylor series according to Eq. (7)-(8).

In Section II we introduce a simplified system of rescaled equations for the reconnection layer. In Section III we present some numerical evidence for the existence of the viscous boundary layer inside the reconnection region. In Section IV we perform the boundary layer analysis and show how the singularity emerges as $\nu \rightarrow 0$. We then present our conclusions in Section V.

\section{THE SYSTEM OF RESCALED EQUATIONS.}

First, it is convenient to simplify our MHD equations by making use of the smallness of the resistivity $\eta$, and to rescale it out of the problem. In other words, we rescale the distances and the fields in the $y$-direction to the corresponding global values (i.e. the length of the layer $L$, the magnetic field just outside of the layer $B_{0}$, and the corresponding Alfvén speed $V_{A}$ ), while rescaling the distances in the $x$-direction and the $x$-components of the velocity and magnetic field to the corresponding Sweet-Parker values:

$$
\begin{array}{cl}
\frac{y}{L} \rightarrow y & \frac{x}{l} \rightarrow x \\
\frac{v_{y}}{V_{A}} \rightarrow v_{y} & \frac{v_{x}}{V_{A} l / L} \rightarrow v_{x} \\
\frac{B_{y}}{B_{0}} \rightarrow B_{y} & \frac{B_{x}}{B_{0} l / L} \rightarrow B_{x} \\
\frac{p}{B_{0}^{2} / 4 \pi} \rightarrow p & \frac{E}{B_{0} V_{A} l / L} \rightarrow E
\end{array}
$$


where $l=l_{S P}=L S^{-1 / 2}$ - is the Sweet-Parker thickness of the current layer, and $S=L V_{A} / \eta$ is the global Lindquist number. The viscosity $\nu$ is now rescaled as $\nu / \eta \rightarrow \nu$.

Now we can write the $z$-component of the Ohm law (1) in the steady state as

$$
E=\frac{\partial^{2} \Psi}{\partial x^{2}}-v_{x} B_{y}+v_{y} B_{x}
$$

Since all the velocities in the $x$-direction are small compared with the Alfvén speed, the $x$-component of the equation of motion (2) just gives us the pressure balance across the current sheet:

$$
\frac{\partial}{\partial x}\left(p+\frac{B_{y}^{2}}{2}\right)=0
$$

which allows us to determine pressure in terms of $B_{y}(x, y)$, once we know the pressure and the magnetic field outside of the reconnection layer. As usual, we set the pressure to zero outside the layer. Also, we take the magnetic field $B_{0 y}(y)$ outside the reconnection layer in the simplest form corresponding to the Syrovatskii current sheet $[7]: B_{0 y}(y)=\sqrt{1-y^{2}}$. Then

$$
p(x, y)=\left(1-y^{2}\right) / 2-\frac{B_{y}^{2}(x, y)}{2}
$$

Finally, we have the $y$-component of the equation of motion (2), with acceleration produced by both the pressure gradient and the magnetic tension, and with the viscous force:

$$
\vec{v} \cdot \nabla v_{y}=y+\vec{B} \cdot \nabla B_{y}+\nu \frac{\partial^{2} v_{y}}{\partial x^{2}}
$$

where we have used the pressure balance Eq. (18).

Let us remark that, when the rescaled viscosity is small $(\nu \ll 1)$, it plays a role of the new small parameter in Eq. (19), and is responsible (as will be shown in Section IV) for the viscous boundary layer inside the main reconnection layer. (Since the physically important viscosity is the viscosity perpendicular to the magnetic field, it is reasonable that it is small compared to $\eta$ or, in the rescaled units, $\nu \ll 1$.)

Now, if one applies the same Taylor expansion procedure as in Section I to the rescaled

equations (16) and (19), one can easily get a result similar to that by Shivamoggi [3,4] and Biskamp $[5,6]$ (note that now we consider $x \rightarrow 0$ on the microscopic scale, i.e. $x \ll l_{S P}$ ). 
From Ohm's law (16) we get:

$$
\begin{gathered}
\Psi_{40}=-2 \Phi_{11} \Psi_{20} \\
\Psi_{22}=2 \Phi_{11} \Psi_{02}
\end{gathered}
$$

And from the equation of motion (19) we get to the lowest order

$$
\Phi_{11}^{2}=1-\Psi_{20} \Psi_{02}+\nu \Phi_{31}
$$

and in the next order in $x$, using (20) and (21):

$$
-4 \Phi_{11} \Psi_{20} \Psi_{02}=\nu \Phi_{51}
$$

Again, if one argues that the RHS vanishes as $\nu \rightarrow 0$, one comes to the same conclusion as in the previous section, namely that $\Psi_{02}=0$, and hence $B_{x}(x=0, y) \sim y^{3}$ near the origin $(0,0)$. Note that finite $\Psi_{02}$ would mean that the separatrices cross at an angle of order of the inverse aspect ratio of the current sheet, i.e. of order $l_{S P} / L \sim S^{-1 / 2} \ll 1$. This angle is small, but still not zero. However, if $\Psi_{02}=0$, then the separatrices have to osculate even on this Sweet-Parker scale, i.e. they cross at an angle which is really equal to zero. The rest of this paper is devoted to showing that $\Psi_{02}$ does not have to be zero even in the inviscous case, in contradiction with the conclusions by Priest and Cowley, Shivamoggi, and Biskamp.

We believe that, by doing this rescaling procedure, we have preserved all the important features of the reconnection problem, in particular, the nature and the main result of Priest's and Cowley's argument outlined in Section I. We shall therefore analyze the system of rescaled equations (16), (19). The main result of our analysis, i.e. the presence of the viscous boundary layer along $x=0$ and the emergence of singular behavior in the limit $\nu \rightarrow 0$, should be true for the system of unrescaled equations (5) and (6) as well, thus invalidating the argument by Priest and Cowley, Shivamoggi, and Biskamp. One should remember, however, that finite $\Psi_{02}$ now means that the angle between the separatrices is finite only on the Sweet-Parker scale, and that the real angle is of order $S^{-1 / 2}$. 


\section{NUMERICAL SIMULATION.}

In order to study the behavior of our system, we ran a series of numerical simulations for different values of the viscosity. The steady state described by the system of rescaled equations (16) and (19), was approached by means of the time-dependent equations:

$$
\begin{gathered}
\frac{\partial \Psi}{\partial t}=\frac{\partial^{2} \Psi}{\partial x^{2}}-v_{x} B_{y}+v_{y} B_{x} \\
\frac{\partial v_{y}}{\partial t}=-\vec{v} \cdot \nabla v_{y}+y+\vec{B} \cdot \nabla B_{y}+\nu \frac{\partial^{2} v_{y}}{\partial x^{2}}
\end{gathered}
$$

with natural unit of time being the Alfvén time $\tau_{A}=L / V_{A}$. The details of the simulation, the numerical scheme, and the boundary conditions will be described later in a subsequent paper.

In our simulation we have found that the system approaches a unique steady state independent of the initial conditions in a few Alfvén times. The steady state electric field was

found to be 1.07 times the Sweet-Parker value $\left(B_{0} V_{A} / \sqrt{S}\right)$. We also found that relationships (20)-(23) are indeed satisfied. However, the coefficient $\Psi_{02}$ approaches a finite value in the limit $\nu \rightarrow 0$, while $\Phi_{51}$ goes to infinity in this limit in such a way that $\nu \Phi_{51}$ in equation (23) remains finite. The coefficient $\Psi_{31}$ has a logarithmic dependence on the viscosity, which will be explained at the end of the next Section. The dependence of the expansion coefficients on the viscosity is summarized in Table 1, and in Figs. $3 a$ and $3 b$. Their behavior suggests a viscous boundary layer, so we performed the boundary layer analysis presented in the next section.

\section{BOUNDARY LAYER ANALYSIS.}

In this section we consider the system of rescaled steady state equations (16), (19) in the small viscosity limit.

Near the origin, let us write the expansion for $\Psi$ up to the second order in $y$ and the expansion for $\Phi$ up to the first order in $y$ in the following forms: 


$$
\begin{gathered}
\Psi(x, y)=\Psi_{20} \frac{x^{2}}{2}+\Psi_{02} \frac{y^{2}}{2}+\Psi_{40} \frac{x^{4}}{24}+\Psi_{22} \frac{x^{2} y^{2}}{4}+\tilde{\Psi}_{1}(x)+\tilde{\Psi}_{2}(x) \frac{y^{2}}{2}+\ldots \\
\Phi(x, y)=\Phi_{11} x y+\tilde{\Phi}(x) y+\ldots
\end{gathered}
$$

Here $\Psi_{20}, \Psi_{02}, \Psi_{40}, \Psi_{22}$, and $\Phi_{11}$ are the values of the corresponding derivatives at the origin $(0,0)$ when the viscosity is set to zero. The functions $\tilde{\Phi}(x), \tilde{\Psi}_{1}(x)$, and $\tilde{\Psi}_{2}(x)$ depend also on $\nu$, and are such that $\tilde{\Psi}_{1}(x) \ll x^{4}, \tilde{\Psi}_{2}(x) \ll x^{2}$, and $\tilde{\Phi}(x) \ll x$ as $x \rightarrow 0, \nu \rightarrow 0$. As we shall see, they vary finitely in the viscous layer that emerges from our equations.

From Ohm's law in the second order in $x$ and the zeroth order in $y$ and $\nu$ we get Eq. (20), and, similarly, in the zeroth order in $x$ and $\nu$ and the second order in $y$ we get Eq. $(21)$.

Now, in the $y$-component of the equation of motion, we can, to linear order in $y$, neglect the $\tilde{\Psi}_{1}, \tilde{\Psi}_{2}$-terms for small $x$. (The neglect of these terms can be justified after the complete solution is obtained). We get

$$
\begin{gathered}
\Phi_{11}^{2}+\Phi_{11}\left[2 \tilde{\Phi}^{\prime}(x)-x \tilde{\Phi}^{\prime \prime}(x)\right]+\left(\tilde{\Phi}^{\prime}(x)\right)^{2}-\tilde{\Phi}(x) \tilde{\Phi}^{\prime \prime}(x)= \\
=1-\Psi_{20} \Psi_{02}+2 \Phi_{11} \Psi_{20} \Psi_{02} x^{2}+\nu \tilde{\Phi}^{\prime \prime \prime}(x)+\text { H.O.T. }
\end{gathered}
$$

where we used Eqs. (20)-(21). Here, H.O.T. stands for "Higher Order Terms".

In the zeroth order in both $x$ and $\nu$ we get

$$
\Phi_{11}^{2}=1-\Psi_{20} \Psi_{02}
$$

which is equivalent to Eq. (22) in the limit $\nu \rightarrow 0$. (Again, here $\Phi_{11}, \Psi_{20}$ and $\Psi_{02}$ are the values of the derivatives $\Phi_{x y}(0,0), \Psi_{x x}(0,0) \Psi_{y y}(0,0)$, respectively, when $\nu=0$. The correction $\Delta \Phi_{11}$ due to a non-zero viscosity will be calculated at the end of this Section.)

Then, in the next order, neglecting the non-linear (and therefore higher order) terms $\left(\tilde{\Phi}^{\prime}(x)\right)^{2}$ and $\tilde{\Phi}(x) \tilde{\Phi}^{\prime \prime}(x)$, we get

$$
2 \tilde{\Phi}^{\prime}(x)-x \tilde{\Phi}^{\prime \prime}(x)=\alpha x^{2}+\nu \frac{1}{\Phi_{11}} \tilde{\Phi}^{\prime \prime \prime}(x)
$$


where $\alpha=2 \Psi_{20} \Psi_{02}$. The last term in Eq. (30) gives rise to a boundary layer related to the small parameter $\nu$.

\section{Outer Region Analysis:}

Outside of the boundary layer, the thickness $\delta$ of which will be estimated later, we can neglect the viscous term:

$$
2 \tilde{\Phi}_{\text {outer }}^{\prime}(x)-x \tilde{\Phi}_{\text {outer }}^{\prime \prime}(x)=\alpha x^{2}
$$

This equation describes the behavior of $\tilde{\Phi}_{\text {outer }}(x)$ for small $x$ (but $x \gg \delta$ ).

First we consider the homogeneous equation $2 \tilde{\Phi}^{\prime}(x)-x \tilde{\Phi}^{\prime \prime}(x)=0$. The general solution of this equation is $\tilde{\Phi}_{h}(x)=a+b x^{3}$. A particular solution of the non-homogeneous equation (31) is $\tilde{\Phi}_{n h}(x)=-(\alpha / 3) x^{3} \ln x$. Thus, the general solution of the non-homogeneous second order differential equation $(31)$ can be written near $x=0$ as

$$
\tilde{\Phi}_{\text {outer }}(x)=-\frac{\alpha}{3} x^{3} \ln x+b x^{3}
$$

where we choose $a=0$ to satisfy the boundary condition $\Phi(0, y)=0$ and hence $\tilde{\Phi}(0)=0$.

Let us remark that the presence of the logarithmic term in (32) is not accidental, but is related to the fact that $x=0$ is a regular singular point for the second order linear differential equation (31). (For more information on how such logarithmic behavior arises near regular singular points see, for example, Ref. [8]).

We now see that in the outer region $\tilde{\Phi}(x) \sim x^{3} \ln x$. This means that we could indeed neglect terms $\left(\tilde{\Phi}^{\prime}\right)^{2}$ and $\tilde{\Phi} \tilde{\Phi}^{\prime \prime}$ in Eq. (28): they give a correction to $\tilde{\Phi}_{\text {outer }}(x)$ of order $x^{5} \ln ^{2} x$.

Also we can now use our estimate for $\tilde{\Phi}_{\text {outer }}(x)$ in order to evaluate $\tilde{\Psi}_{1}$ and $\tilde{\Psi}_{2}$ in the outer region. Substituting expansions (26)-(27) into Ohm's law (16), and considering terms of zeroth order in $y$, we get Eq. (20) to the lowest order in $x$ (i.e. balancing terms of order $x^{2}$, and in the next order we get

$$
\tilde{\Psi}_{1, \text { outer }}^{\prime \prime}(x)=-\Psi_{20} x \tilde{\Phi}_{\text {outer }}(x)
$$

Similarly, by looking at quadratic in $y$ terms in Ohm's law, we get Eq. (21) to the lowest 
order in $x$, and in the next order in $x$ we get

$$
\tilde{\Psi}_{2, \text { outer }}^{\prime \prime}(x)=2 \Psi_{02} \tilde{\Phi}_{\text {outer }}^{\prime}(x)
$$

Then, using (32) we can estimate $\tilde{\Psi}_{1, \text { outer }} \sim x^{6} \ln x, \tilde{\Psi}_{2, \text { outer }} \sim x^{4} \ln x$, and we see that the contribution from these terms to the RHS of the equation of motion is indeed small. The analysis of the corrections due to these higher order terms in Eq. (28) shows that they give a correction to $\tilde{\Phi}_{\text {outer }}(x)$ of order $x^{5} \ln x$. Thus, the next order terms in the expansion of $\tilde{\Phi}(x)$ near $x=0$ in the outer region are of order $x^{5} \ln ^{2} x$.

\section{Inner Region Analysis:}

Inside the viscous boundary layer let us rescale our variable $x$ once more:

$$
x \rightarrow \hat{x}=\frac{x}{\delta}
$$

where $\delta=\delta(\nu)$ is the characteristic thickness of the boundary layer. Also, let us write:

$$
\tilde{\Phi}(x)=g(\nu) \phi(\hat{x})
$$

The viscous term becomes important inside the boundary layer, and Eq. (30) is now written as

$$
2 \frac{g}{\delta} \phi^{\prime}(\hat{x})-\frac{g}{\delta} \hat{x} \phi^{\prime \prime}(\hat{x})=\alpha \delta^{2} \hat{x}^{2}+\nu g(\nu) \delta^{-3} \phi^{\prime \prime \prime}(\hat{x}) \frac{1}{\Phi_{11}}
$$

Now the term with the highest order derivative is the viscous term. The point $\hat{x}=0$ is now just a regular point and $\phi(\hat{x})$ is an analytic and odd function of $\hat{x}$.

The appropriate balance in Eq. (37) corresponds to

$$
\delta=\nu^{1 / 2} \quad g(\nu)=\delta^{3}=\nu^{3 / 2}
$$

Again we see that the non-linear terms $\left(\tilde{\Phi}^{\prime}\right)^{2}$ and $\tilde{\Phi} \tilde{\Phi}^{\prime \prime}$ in Eq. (28) are small (of order $\left.g^{2} / \delta^{2}=\nu^{2} \ll g / \delta\right)$. The same is true for the contribution from the higher order terms in the expansion (26) (i.e. the $\tilde{\Psi}$-terms) to the forces on the RHS of Eq. (28). Thus we see that Eq. (30) holds also in the inner region (i.e. inside the boundary layer). 
Using relationships (38) in Eq. (37) we get

$$
2 \phi^{\prime}(\hat{x})-\hat{x} \phi^{\prime \prime}(\hat{x})=\alpha \hat{x}^{2}+\beta \phi^{\prime \prime \prime}(\hat{x})
$$

where $\beta=1 / \Phi_{11}$.

In order to solve this equation, we first introduce $t=\hat{x} / \sqrt{\beta}, p(t) \equiv \phi^{\prime}(\hat{x}(t))$, reducing Eq. (39) to

$$
p^{\prime \prime}(t)+t p^{\prime}(t)-2 p(t)=-\alpha \beta t^{2}
$$

The general solution of this non-homogeneous equation is

$$
p(t)=p_{1}(t) C_{1}(t)+p_{2}(t) C_{2}(t)
$$

where

$$
p_{1}(t)=1+t^{2} \quad \text { and } \quad p_{2}(t)=\left(1+t^{2}\right) \int_{\infty}^{t} \frac{e^{-t^{2} / 2}}{\left[1+t^{2}\right]^{2}} d t
$$

are the two independent solutions of the homogeneous equation $p^{\prime \prime}(t)+t p^{\prime}(t)-2 p(t)=0$.

The coefficients $C_{1}(t), C_{2}(t)$ satisfy the following system of differential equations:

$$
\begin{gathered}
C_{1}^{\prime}(t) p_{1}(t)+C_{2}^{\prime}(t) p_{2}(t)=0 \\
C_{1}^{\prime}(t) p_{1}^{\prime}(t)+C_{2}^{\prime}(t) p_{2}^{\prime}(t)=-\alpha \beta t^{2}
\end{gathered}
$$

Hence,

$$
\begin{gathered}
C_{1}^{\prime}(t)=\alpha \beta t^{2}\left(1+t^{2}\right) e^{t^{2} / 2} \int_{\infty}^{t} \frac{e^{-t^{2} / 2}}{\left[1+t^{2}\right]^{2}} d t \\
C_{2}^{\prime}(t)=-\alpha \beta t^{2}\left(1+t^{2}\right) e^{t^{2} / 2}
\end{gathered}
$$

The next step is to examine the asymptotic behavior of $\phi(\hat{x})$ for $\hat{x} \rightarrow \infty$ in order to see how the (non-analytic) log-like behavior (32) emerges in the outer region.

Consider $I(t) \equiv \int_{\infty}^{t} \frac{e^{-t^{2} / 2}}{\left(1+t^{2}\right)^{2}} d t$ in the limit $t \rightarrow \infty$. By taking the integral by parts, we see that:

$$
I(t)=-t^{-5} e^{-t^{2} / 2}+O\left(t^{-7} e^{-t^{2} / 2}\right)
$$


Then

$$
C_{1}(t)=C_{10}-\alpha \beta \ln (t)+O\left(t^{-2}\right), \quad t \rightarrow \infty
$$

where $C_{10}$ is the arbitrary constant of integration.

Also we can estimate

$$
C_{2}(t)=-\alpha \beta t^{3} e^{t^{2} / 2}+O\left(t e^{t^{2} / 2}\right), \quad t \rightarrow \infty
$$

Substituting these $C_{1}(t)$ and $C_{2}(t)$ into Eq. (41) we get

$$
p(t \rightarrow \infty)=-\alpha \beta t^{2} \ln (t)+C_{10} t^{2}+O(\ln t)
$$

Then,

$$
\phi(\hat{x} \rightarrow \infty)=-\frac{\alpha}{3} \hat{x}^{3} \log \hat{x}+C_{10}^{*} \hat{x}^{3}+O(\hat{x} \ln \hat{x})
$$

where

$$
C_{10}^{*}=\frac{1}{3}\left(\alpha / 3+C_{10} \Phi_{11}-\frac{\alpha}{2} \ln \Phi_{11}\right)
$$

Then,

$$
\tilde{\Phi}_{\text {inner }}(x \gg \delta)=g(\nu) \phi(\hat{x})=-\frac{\alpha}{3} x^{3} \ln x+\frac{\alpha}{3} x^{3} \ln \delta+C_{10}^{*} x^{3}+\nu O(x \ln \hat{x})
$$

This expression must match $\tilde{\Phi}_{\text {outer }}(x)=-\frac{\alpha}{3} x^{3} \ln x+b x^{3}$ (see Eq. (32)). Therefore,

$$
C_{10}=\left[3 b-\frac{\alpha}{2} \ln \nu-\frac{\alpha}{3}+\frac{\alpha}{2} \ln \Phi_{11}\right] / \Phi_{11}
$$

Because $\alpha, b$, and $\Phi_{11}$ come from the outer solution, they are independent of $\nu$. Therefore, in the limit of small $\nu$, we can estimate $C_{10}$ as

$$
C_{10}=-\frac{\alpha}{2 \Phi_{11}} \ln \nu+O(1)
$$

Next, let us consider our solution (41) in the limit $\hat{x} \rightarrow 0$, and see what we can learn about the derivatives $\Phi_{31}=\Phi_{x x x y}(0,0)$ and $\Phi_{51}=\Phi_{x x x x y}(0,0)$. 
For $t \rightarrow 0$,

$$
C_{1}(t)=C_{1}(0)-\alpha \beta \Gamma t^{3} / 3+\alpha \beta t^{4} / 4+O\left(t^{6}\right)
$$

where $\Gamma=\int_{0}^{\infty} \frac{e^{-t^{2} / 2}}{\left(1+t^{2}\right)^{2}} d t$

Also,

$$
C_{2}(t)=C_{2}(0)-\frac{\alpha \beta}{3} t^{3}+O\left(t^{5}\right)
$$

Then, from the fact that $p(t)$ must be an even function of $t$, and therefore $C_{2}(0)=0$, we get

$$
p(t \rightarrow 0)=C_{1}(0)+C_{1}(0) t^{2}-\alpha \beta t^{4} / 12+\ldots
$$

Then, using $\phi(0)=0$,

$$
\tilde{\Phi}_{\text {inner }}(x \ll \delta)=C_{1}(0) \nu x+C_{1}(0) \Phi_{11} \frac{x^{3}}{3}-\nu^{-1} \alpha \beta \Phi_{11}^{2} \frac{x^{5}}{60}+\ldots
$$

Now, $C_{1}(0)$ in general also depends on $\nu$. It is related to the integration constant $C_{10}$ : $C_{1}(0)=C_{10}+O(1)$. Thus, when $\nu$ is small, we get

$$
C_{1}(0) \sim C_{10}(\nu) \sim-\frac{\alpha}{2 \Phi_{11}} \log \nu=-\frac{\Psi_{20} \Psi_{02}}{\Phi_{11}} \log \nu
$$

Then, from Eq. (56) we see that

$$
\begin{gathered}
\Delta \Phi_{11}=\left.\frac{\partial \tilde{\Phi}}{\partial x}\right|_{x=0} \sim C_{1}(0) \nu \sim-\frac{\Psi_{20} \Psi_{02}}{\Phi_{11}} \nu \ln \nu \ll \Phi_{11}=O(1) \\
\Phi_{31}=-\alpha \log \nu+O(1)=-2 \Psi_{20} \Psi_{02} \log \nu+O(1) \\
\Phi_{51}=-4 \Phi_{11} \Psi_{20} \Psi_{02} \nu^{-1}
\end{gathered}
$$

Therefore, inside the boundary layer Eq. (23) is indeed satisfied, even when $\Psi_{02} \neq 0$ as $\nu \rightarrow 0$.

In Figs. $3 a$ and $3 b$ we give the comparison of our numerical results with the analytical predictions (59)-(60) for $\Phi_{31}$ and $\Phi_{51}$, for different values of viscosity. In order to calculate 
$\Phi_{31}$ and $\Phi_{51}$ from Eqs. (59)-(60), we have used the values of the coefficients $\Phi_{11}, \Psi_{20}$, and $\Psi_{02}$ obtained in the numerical simulation with $\nu=0.005$ ( the smallest value of viscosity in our simulations).

We see that the agreement between (59)-(60) and the numerically obtained values of $\Phi_{31}$ and $\Phi_{51}$ is very good, and it improves with decreasing viscosity.

\section{DISCUSSION AND CONCLUSIONS.}

In this paper we present a boundary layer analysis near the X-type neutral point at the center of the reconnection layer in incompressible resistive MHD. First we show that, in the case of zero viscosity, the Taylor expansion procedure employed by Priest and Cowley $[1,2]$, Shivamoggi [3,4], and Biskamp [5,6] is not justified. The solution of the MHD equations (16) and (19) with $\nu=0$ is given in Section IV by our outer solution (see equations (27) and (32) for the velocity stream function $\Phi$, and by equations (26) and (33)-(34) for the magnetic flux function $\Psi$ ). It is clear that $\Phi$ and $\Psi$ do not have a Taylor expansion near $x=0$, contrary to the assumption of Refs. [1]-[6]. Thus we find that the conclusion that $\Psi_{02}=0$ when $\nu=0$, and hence that the magnetic separatrices have to osculate at the $\mathrm{X}$-point in this case, is not correct.

Then we consider the case of small but finite viscosity. We show, both numerically and analytically, that, as the viscosity $\nu$ becomes very small, some of the higher derivatives of the stream function, particularly $\Phi_{51}$, become very large. The product $\nu \Phi_{51}$ remains finite, and from Eq. (23) it follows that $\Psi_{02}$ does not have to become zero. In general, it stays finite (on the Sweet-Parker scale - see discussion at the end of Section II) as $\nu \rightarrow 0$. The value of $\Psi_{02}$ (as well as the values of some other expansion coefficients, such as $\Psi_{20}$ and $\left.\Phi_{11}\right)$ can not be determined from the analysis in the vicinity of the X-point only. In fact, this value is determined by the solution of the total problem concerning the entire resistive reconnection layer, in particular by the boundary conditions for the reconnection

layer. These boundary conditions are represented by the magnetic field $B_{0 y}(y)$ just outside 
of the reconnection layer [9]. In our numerical simulation we have studied the case when $B_{0 y}(y)=B_{0} \sqrt{1-y^{2} / L^{2}}$, which is a generic form for a typical Syrovatskii-like current sheet [7]. The value of $\Psi_{02}$ was found to be equal to -0.64 (see Table I).

We would like to point out that it might be not impossible for one to find some special case of these boundary conditions corresponding to $\Psi_{02}=0$, in which case the separatrices would osculate. In general, however, this is not so, and $\Psi_{02} \neq 0$.

It is worthwhile to mention that in this paper we have considered only the boundary layer (and the corresponding non-analytic behavior emerging in the limit $\nu \rightarrow 0$ ) only in the $x$ direction. Our analysis assumes that the behavior in the $y$-direction is regular, at least up to the required orders in $y$ (i.e. linear in the equation of motion (for function $\Phi$ ) and quadratic for $\Psi)$. This is justified by the results of our numerical simulation. However, if we went to higher orders in $y$, we would probably encounter non-analytic, log-like dependence of $\Phi$ and $\Psi$ on $y$. Indeed, in the physically most interesting limit of infinitely large Lundquist number, which we consider here, the resistivity acting in the $y$-direction in our rescaled equations is negligible. Since we consider the limit when the viscosity is small compared with the resistivity, we then have to let the viscosity acting in the $y$-direction also go to zero in the rescaled equations. Then, since $V_{y}(y=0, x)=0$, we see that, for both Ohm's law (16) and the equation of motion (19), $y=0$ is a regular singular point in the $y$-direction. Therefore, we should expect some non-analytic behavior in the $y$-direction (as well as in the $x$-direction). Apparently, however, because the $x$ - and $y$-directions enter in a very non-symmetric way in our rescaled equations, such a non-analytic behavior exhibits itself only in sufficiently high orders in $y$. In addition, it is not easy to recognize such a non-analytic behavior in $y$ in a numerical simulation, because there is no qualitative feature associated with it as the crossing of the separatrices at a finite angle is associated with the non-analyticity in the $x$-direction.

If we look into a situation with finite Lundquist number $S$, we will have to consider the original unrescaled equations (5)-(6) - our rescaling procedure of Section II does not work (note that Priest, Cowley, Shivamoggi, and Biskamp do not actually assume large $S$ in 
their analyses). In this case, as we consider the limit of small (compared with the resistivity) viscosity, we should expect boundary layers along both the $x$ - and $y$-axes, because these axes are the separatrices for the velocity field. These boundary layers cross at the stagnation point at a $90^{\circ}$ angle. The analysis becomes rather complicated, because of the larger number of terms involved, and also because of a possible interaction between the two boundary layers. Nevertheless, the qualitative picture will remain the same: some of the higher derivatives will be very large inside the boundary layers, and outside of the boundary layers the $\Psi$ and $\Phi$ functions will have non-regular behavior in the limit $\nu \rightarrow 0$. Thus, we expect that the conclusion that $\Psi_{02}=0$ will fail again, and the separatrices will cross at a non-zero angle. In any event, we can conclude that the argument leading to $\Psi_{02}=0$ in unrescaled equations can not be rigorous, because the same argument could be used to imply $\Psi_{02}=0$ in the rescaled equations.

\section{ACKNOWLEDGMENTS.}

We are grateful to Dieter Biskamp, Steve Cowley, Hantao Ji, B.C.Low, and Masaaki Yamada for a number of helpful discussions.

This work was supported by Charlotte Elizabeth Procter Fellowship, by the Department of Energy Contract No. DE-AC02-76-CHO-3073, and by NASA's Astrophysical Program under Grant NAGW2419.

[1] E.R. Priest and S.W.H. Cowley, J. Plasma Physics, 14, part II, 271-282 (1975).

[2] Cowley, S.W.H., J. Plasma Phys. 14, part III, 475-490 (1975).

[3] B.K. Shivamoggi, J. Plasma Physics 31, 333-335 (1985).

[4] B.K. Shivamoggi, Phys. Reports, 127, 101 (1985) 
[5] D. Biskamp, "Nonlinear Magnetohydrodynamics" (Cambridge University Press, Cambridge, 1993), p.147.

[6] D. Biskamp, Phys. Reports, 237, 179 (1994)

[7] S.I. Syrovatskii, Sov. Phys. JETP, 33, 933 (1971).

[8] Bender, Carl M., and Orszag, Steven A., “Advanced Mathematical Methods for Scientists and Engineers", McGrow-Hill Book Company, New York 1978, p. 63, 68-76. Also see F.W.J.Olver, “Asymptotics and Special Functions", N.Y., L.: Academic Press, 1974.

[9] Dmitri A. Uzdensky and Russell M. Kulsrud, Phys. Plasmas, 4, 3960 (1997). 


\begin{tabular}{|c|c|c|c|c|c|c|}
\hline \hline Viscosity $\nu$ & $\Phi_{11}$ & $\Psi_{20}$ & $\Psi_{02}$ & $\Phi_{31}$ & $\Phi_{51}$ & $\nu \Phi_{51}$ \\
\hline 0.04 & 1.237 & 1.078 & -0.648 & -4.12 & 84.6 & 3.384 \\
\hline 0.02 & 1.261 & 1.076 & -0.644 & -5.06 & 173.1 & 3.462 \\
\hline 0.01 & 1.276 & 1.075 & -0.643 & -6.03 & 352.4 & 3.524 \\
\hline 0.005 & 1.286 & 1.075 & -0.642 & -7.01 & 713.8 & 3.569 \\
\hline \hline
\end{tabular}

TABLE I. Results of the numerical simulations: expansion coefficients corresponding to several different values of the viscosity. 


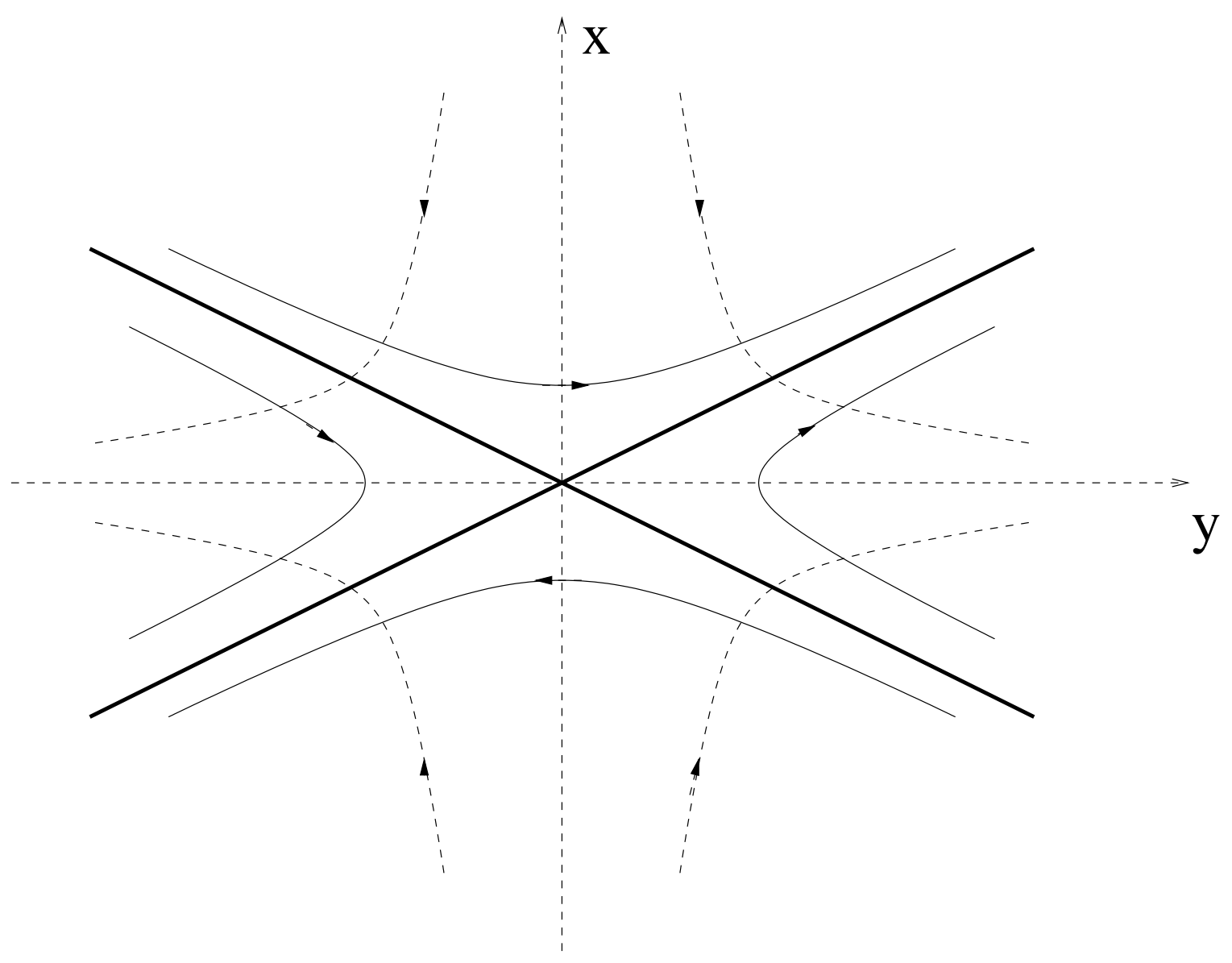

FIG. 1. The X-point configuration with magnetic separatrices crossing at a finite angle at the neutral point. Solid lines represent magnetic field lines, dashed lines represent the velocity streamlines. Heavy solid lines represent magnetic separatrices. 


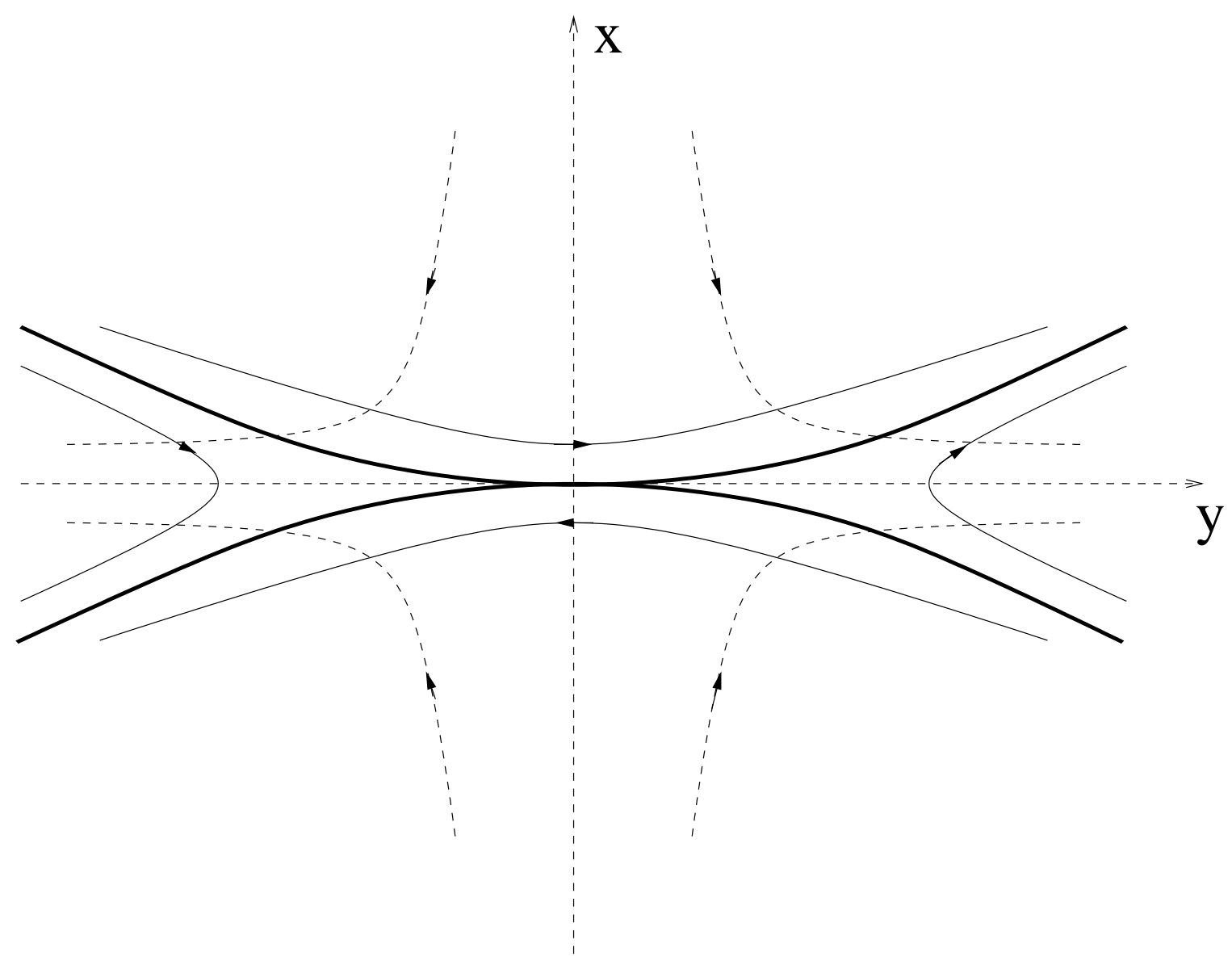

FIG. 2. The X-point configuration with magnetic separatrices osculating at the neutral point. 


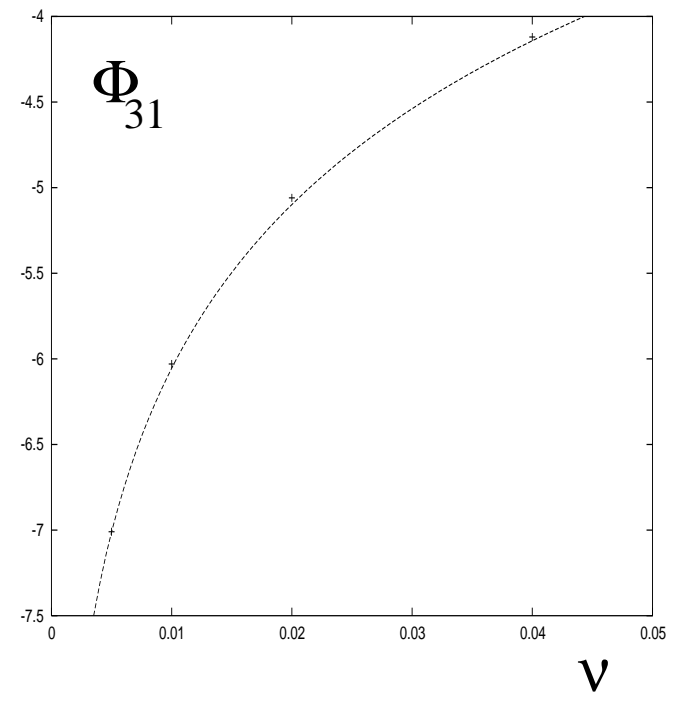

(a)

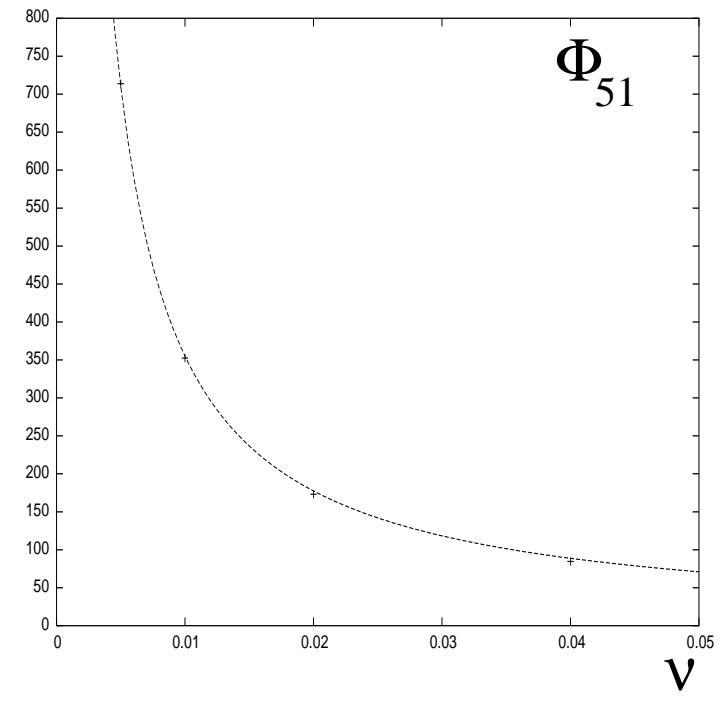

(b)

FIG. 3. Numerical and analytical results for the dependence of the coefficients $\Phi_{31}$ (Figure a) $\Phi_{51}$ (Figure $b$ ) and on the viscosity $\nu$. Continuous lines represent the analytical predictions $\Phi_{31}(\nu)=-2 \Psi_{20} \Psi_{02} \log \nu+0.30$, in accordance with Eq. (59), and $\Phi_{51}(\nu)=-4 \Phi_{11} \Psi_{20} \Psi_{02} / \nu$, in accordance with Eq. (60). (The values of $\Phi_{11}, \Psi_{20}$ and $\Psi_{02}$ used in Eqs. (59)-(60) are taken from the results of the numerical simulations. The arbitrary additive constant $0.30 \mathrm{in} \mathrm{Eq.} \mathrm{(59)} \mathrm{is} \mathrm{chosen}$ to fit the numerical value of $\Phi_{31}$ at the point $\nu=0.005$.) The values of $\Phi_{31}$ and $\Phi_{51}$ obtained in the numerical simulations for four different values of viscosity $(\nu=0.04, \nu=0.02, \nu=0.01$, and $\nu=0.005)$ are given by dots. 